\title{
Wann macht der Job tatsächlich krank?
}

$11 \%$ aller Fehltage gehen auf eine psychische Erkrankung zurück, hat eine aktuelle Studie der Bundespsychotherapeutenkammer (BPtK) ergeben. Damit hat sich ihre Zahl seit 1990 fast verdoppelt. Welche Rolle die heutigen Arbeitsbedingungen dabei spielen, erklärt Werner Gross vom

\begin{tabular}{|l|l|} 
Nachgefragt & $\begin{array}{l}\text { bei Werner Gross } \\
\text { "Beschäftigte } \\
\text { im Sozial- und } \\
\text { Gesundheits- } \\
\text { wesen besonders } \\
\text { gefährdet“ }\end{array}$ \\
\hline
\end{tabular}
Psychologischen Forum Offenbach.

\begin{abstract}
MMW: Was sind die häufigsten arbeitsbedingten psychischen Erkrankungen?

Gross: Es beginnt meist mit einer Belastungsstörung, die sich aufgrund der Angst um den Job entwickelt. Später folgen Depressionen, Suchterkrankungen und psychosomatische Erkrankungen, abhängig von der Veranlagung des Patienten.
\end{abstract}

MMW: Worin sehen Sie die Ursachen für die Zunahme dieser Erkrankungen?

Gross: Viele Leute leiden unter Zeitdruck, und auch die Qualitätsanforderungen werden immer höher, sodass kaum noch Zeit zum Durchatmen und für ein Schwätzchen bleibt. Das wäre aber für ein gutes Klima im Team notwendig. Hinzu kommt die ständige Erreichbarkeit über Handy und E-Mail. Viele Arbeitnehmer laufen wie ein Karussell, das sich immer schneller dreht.

Für problematisch halte ich auch die mangelnde Wertschätzung. In Deutschland ist es schon als gut anzusehen, wenn man nicht kritisiert wird. In anderen Ländern gibt es dagegen eher eine Lobkultur.

MMW: Gibt es Berufsgruppen, die besonders gefährdet sind?

Gross: Inzwischen gibt es kaum noch eine Berufsgruppe, die von der "Arbeitsverdichtung" ausgespart wird. Besonders gefährdet für psychische Erkrankungen sind aber die Mitarbeiter im Dienstleistungssektor, in Callcentern und Hotlines sowie Beschäftigte im Sozial- und Gesundheitswesen.

MMW: Woran ist zu erkennen, dass jemand nicht nur unter Stress steht, sondern dass die Arbeit ihn tatsächlich krank macht?

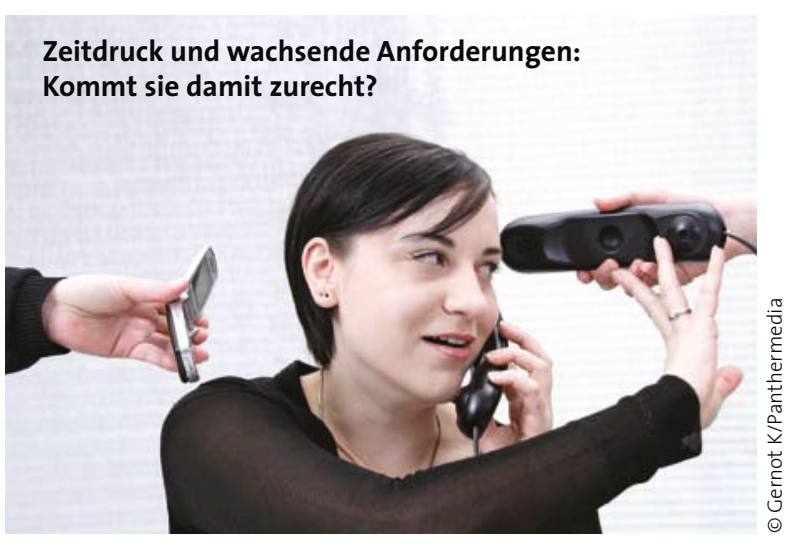

kann, dass er damit klarkommt. Man muss sich überlegen, wie man die Arbeit effizient gestalten kann, ohne dass man sich kaputt macht (,Jobcrafting“). Eine andere Möglichkeit ist die Jobrotation, bei der die Mitarbeiter eines Unternehmens ihre Aufgabengebiete immer wieder tauschen, sodass es keine einseitigen Arbeitsbelastungen gibt. Auch Gesundheitszirkel und regelmäßige Teambesprechungen können helfen. Außerdem kann

Gross: Eine schnelle Diagnose ist hier nicht möglich, weil es sich immer um ein multifaktorielles Geschehen handelt. Der Arzt muss sich die Person auf der somatischen Ebene anschauen, z.B. die körperliche Konstitution und den Schlaf. Gleichzeitig muss er nach der psychischen Verfassung fragen. Dabei kommt man nicht umhin, sich dezidiert die Arbeitssituation beschreiben zu lassen. Wichtige Fragen sind z.B. „Mit welchem Gefühl gehen Sie morgens zur Arbeit?“, ,W Wie sieht der Arbeitsablauf aus?", „Haben Sie noch das Gefühl zu leben oder funktionieren Sie nur noch?“.

MMW: In welchen Situationen würden Sie einen Arbeitsplatzwechsel empfehlen? Gross: Wenn die Atmosphäre zwischen dem Vorgesetzten und/oder im Team so vergiftet ist, dass man seine Arbeit nicht mehr angemessen ausführen kann oder die Menge der Arbeit nicht mehr ohne massive körperliche bzw. seelische Belastungen zu bewältigen ist.

MмW: Wie könnte der Ausweg aussehen, wenn ein Arbeitsplatzwechsel nicht infrage kommt?

Gross: Es gibt einige Möglichkeiten, wie sich jeder seinen Job so zurechtbiegen jeder Einzelne an sich selbst arbeiten, z.B. mithilfe des 5-Säulen-Modells (s. Tabelle 1). Dabei sollte man nicht nur darauf achten, dass das Arbeitsleben in Ordnung ist, sondern auch körperliche Konstitution, Familie und soziale Beziehungen.

MMW: Wie kann eine Therapie helfen? Gross: Eine Psychotherapie ist kein Allheilmittel, aber sie bietet die Chance, sich die Situation bewusst zu machen, sodass man sie beeinflussen kann. Dabei muss man herausfinden, was geändert werden kann, und womit man leben muss. Psychopharmaka können unterstützend eingesetzt werden, sind aber keine alleinige Lösung.

- Interview: Dr. Judith Neumaier

\section{Tabelle 1}

\section{5-Säulen-Modell}

(1) Arbeit, Leistung, Zeitstruktur

(2) Partnerschaft, Familie

(3) Körper, Gefühle, Gesundheit, Liebe, Sexualität

4 Soziale Beziehungen, Freundeskreis

(5) Sinnsystem

nach W. Gross ,... aber nicht um jeden Preis“, Kreuz Verlag 2010 